\title{
Lenvatinib and other tyrosine kinase inhibitors for the treatment of radioiodine refractory, advanced, and progressive thyroid cancer
}

This article was published in the following Dove Press journal:

OncoTargets and Therapy

20 October 2016

Number of times this article has been viewed

Loredana Lorusso'
Letizia Pieruzzi'
Agnese Biagini'
Elena Sabini'
Laura Valerio'
Carlotta Giani'
Paolo Passannanti'
Benedetta Pontillo-Contillo²
Valentina Battaglia'
Salvatore Mazzeo'
Eleonora Molinaro'
Rossella Elisei'
'Endocrine Unit, Department of
Clinical and Experimental Medicine,
'Division of Diagnostic and
Interventional Radiology, University
of Pisa, Pisa, Italy

Correspondence: Rossella Elisei Endocrine Unit, Department of Clinical and Experimental Medicine, University Hospital, Via Paradisa, 2, 56/24 Pisa, Italy Tel +39050544723

Fax +39050 578772

Email rossella.elisei@med.unipi.it

\begin{abstract}
Lenvatinib is a small oral molecule able to inhibit three of the extracellular and intracellular molecules involved in the modulation of angiogenesis and lymphangiogenesis: vascular endothelial growth factor receptor 1-3, fibroblast growth factor receptor $1-4$, and platelet-derived growth factor receptor alpha. Since it is also able to inhibit the REarranged during Transfection oncogene and the protooncogene $c-K I T$, this drug can also be used to control tumor cell proliferation. The maximum tolerated dose, as demonstrated in Phase I studies, is $25 \mathrm{mg}$ daily. The drug is rapidly absorbed with maximum concentrations achieved within 3 and 5 hours after administration in fasting and nonfasting treated patients, respectively. The most common adverse events, reported in Phase I study and confirmed in the subsequent Phase II and III studies, are hypertension, proteinuria, and gastrointestinal symptoms such as nausea, diarrhea, and stomatitis. In Phase I studies, efficacy of lenvatinib in solid tumors was demonstrated, and these encouraging results have led to the development of a Phase II study using lenvatinib in advance radioiodine-refractory differentiated thyroid cancer (DTCs) patients. Since an overall response rate of 50\% was reported, this study also confirmed the efficacy of lenvatinib in DTCs patients with an acceptable toxicity profile. Recently, a Phase III study in patients with DTCs (SELECT study) demonstrated the lenvatinib efficacy in prolonging progression-free survival with respect to the placebo (18.3 vs 3.6 months; $P<0.001$ ). Although there was no statistically significant difference in the overall survival of the entire group, this result was observed when the analysis was restricted to both the follicular histotype and the group of senior patients ( $>65$ years). The study confirmed that the most common side effects of this drug are hypertension, diarrhea, decreased appetite, weight loss, nausea, and proteinuria. In this review, we report the results of the main studies on lenvatinib efficacy in patients with advanced and progressive thyroid cancer, mainly in DTCs but also in medullary and anaplastic thyroid cancer. We also compared the efficacy of lenvatinib with that of other tyrosine kinase inhibitors, mainly sorafenib, already tested in the same type of patient population.
\end{abstract}

Keywords: lenvatinib, E7080, tyrosine kinase inhibitor, radioiodine refractory thyroid cancer

\section{Introduction}

Thyroid cancer (TCs) is a rare human malignancy, but it is the most common endocrine neoplasia and the cancer with the greatest increasing rate of incidence. In 2015, it was estimated that there were 62,450 new cases of TCs and an estimate of 1,950 deaths from this disease. ${ }^{1}$

According to the degree of cell differentiation and their ability to maintain the typical features of follicular cells, TCs are distinguished into well-differentiated (DTCs), poorly differentiated, and anaplastic (ATC), which represent 85\%, 5\%, and 
$3 \%$ of the entire series of TCs, respectively. The remaining small percentage is represented by medullary thyroid cancer (MTC), which originates from neural crest C cells (5\%) and other rare histotypes such as lymphoma, sarcoma, and squamous cell carcinoma (2\%).

DTCs are further distinguished into papillary (PTC) and follicular (FTC) carcinomas. In both cases, despite the malignant transformation, the tumoral cells maintain the typical features of "normal" thyroid cells, such as the thyroid-stimulating hormone (TSH) growth dependence and the ability to synthesize and secrete thyroglobulin and to take up iodine. These features represent the rationale for the management and treatment of DTCs patients who are treated first by surgery (total or near-total thyroidectomy) with or without cervical lymphadenectomy, and when required, with radioiodine (131-I) both for the postsurgical thyroid remnant ablation and metastatic lesions. ${ }^{2}$ The TSH suppression through mildly elevated dosages of levotyroxine (L-T4) is part of the treatment in DTCs, especially during the first years after surgery and until evidence of clinical remission. ${ }^{3}$ Less evidence of TSH suppression efficacy is available in dedifferentiated TCs since they lose the expression of thyroid-specific genes, including that of the TSH receptor. ${ }^{4}$

Different from ATC and DTCs that are almost invariably lethal, the survival of DTCs patients is $96.4 \%$ at 35 years. ${ }^{5}$ However, during the long-term follow-up, about $20 \%$ of DTCs patients show a local recurrence (thyroid bed or cervical lymph nodes) and/or distant metastasis (mainly lung and/or bone). ${ }^{6}$ Also in these cases, the prognosis of DTCs patients can be favorable if the metastatic lesions are still 131-I avid, but when 131-I refractoriness has developed, the 10 -years overall survival rate drops to $<10 \%{ }^{7}$

To define a DTCs as a radioactive iodine refractory (RAI-R) tumor, it should fulfill at least one of the following criteria: 1) the malignant/metastatic tissue is unable to take up iodine and the post-131-I whole body scan is negative; 2) the malignant/metastatic tissue, previously able to take up 131-I, loses this ability over time; 3) 131-I up-take is present only in some lesions but not in others; and 4) the disease progresses despite its ability to take up 131-I. ${ }^{8}$ In this subgroup of advanced and RAI-R DTCs patients, other treatment options apart from 131-I administration should be considered as indicated in the current guidelines. ${ }^{8}$

Whenever possible, a local treatment is indicated such as surgery, external beam radiation, and percutaneous ultrasound-guided treatments including radiofrequency or laser or ethanol ablation. These treatments should be considered any time the disease is represented by a well-localized and small-volume metastatic lesion. ${ }^{9-12}$
So far, no systemic therapies have been demonstrated to be effective for the disease control in DTCs patients with metastatic, rapidly progressive, symptomatic, and/or imminently threatening disease. Although the evidence is of very limited results, to date doxorubicin remains the single most effective and approved cytotoxic chemotherapy for the treatment of these patients with a recommended dosage of $60-75 \mathrm{mg} / \mathrm{m}^{2}$ every $3-4$ weeks.

In 2008, motesanib diphosphate, a tyrosine kinase inhibitor (TKI), showed the ability to induce partial response (PR) in patients with progressive advanced or metastatic RAI-R DTCs. ${ }^{13}$ After this first experience, several other studies with other TKIs have been conducted.

The antitumoral activity of TKIs is related to their ability to block tyrosine-kinase receptors (TKRs) by competing with ATP at its binding site. As shown in Figure 1, TKRs are trans-cell-membrane receptors that, when activated by their ligands, are able to transduct extracellular signals to the cytoplasm through mitogen-activated protein kinase and phosphatidylinositol-3 kinase/Akt/mammalian target of rapamycin pathways. A somatic or germline mutation located in the DNA coding for one of these TKRs or in one of the central mediators of these pathways can determine a constitutive activation of the intracellular signaling and cause an uncontrolled cell proliferation, cell dedifferentiation, and reduction of apoptosis. Almost $40 \%-50 \%$ of DTCs harbors a point mutation of $R A F$ (in particular, $B-R A F$ ), while $20 \%-25 \%$ is characterized by the presence of a REarranged during Transfection (RET) rearrangement (RET/PTC1-3); a smaller but not negligible percentage of DTCs, mainly FTC, has an $R A S$ point mutation or $P A X 8 / P P A R \gamma$ rearrangement. ${ }^{14-17}$ Moreover, DTCs, as well as many other human tumors, has mutations or overexpression of some receptors such as vascular endothelial growth factor receptor 1-3 (VEGF-R 1-3), fibroblast growth factor receptor 1-4 (FGF-R 1-4), and platelets-derived growth factor receptor alpha (PDGF-R $\alpha$ ). The TKI can exert a block of these receptors, thus limiting angiogenesis and lymphangiogenesis. ${ }^{18,19}$

At present, two different TKIs, lenvatinib (Lenvima ${ }^{\circledR}$; Eisai Co Ltd, Tokyo, Japan) and sorafenib (Nexavar ${ }^{\circledR}$; Bayer Health Pharmaceutical, Leverkusen, Germany), recently approved by both US Food and Drug Administration and European Medicine Agency, can be used for the treatment of RAI-R DTCs. In addition, another TKI, vandetanib, is under Phase III investigation in patients with RAI-R and progressive DTCs.

In this review, the main studies on lenvatinib efficacy in patients with TCs are reported, and the possible advantages of this drug with respect to other TKIs, mainly sorafenib, are also discussed. 


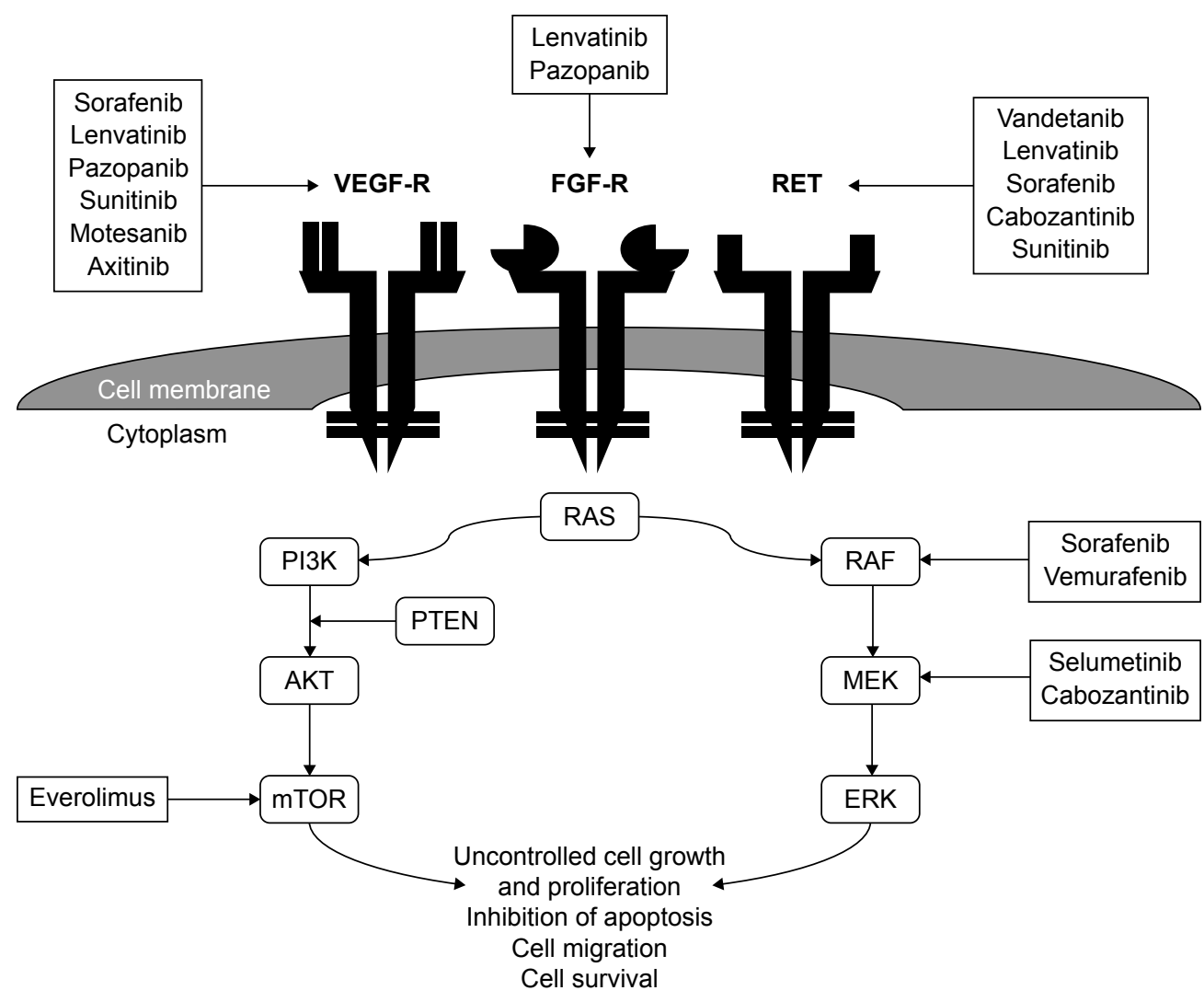

Figure I MAPK and PI3K-AKT-mTOR signaling cascades involved in the development and progression of thyroid cancer after TKI receptor(s) activation.

\section{Lenvatinib}

\section{Drug chemistry and activity}

Lenvatinib (E7080; 4-[3-chloro-4-(N'-cyclopropylureido) phenoxy] 7-methoxyquinoline-6-carboxamide mesylate) is an oral drug blocking several TKRs and was developed by Eisai Co, Ltd. It is able to inhibit VEGF-R 1-3, FGF-R $1-4$, and PDGF-R $\alpha$, thus interfering with angiogenesis and lymphangiogenesis. In parallel, lenvatinib is able to control the tumor cell proliferation through its ability to inhibit $R E T$ oncogene and the oncogene $c-K I T .^{20-22}$ The most important difference between this drug and other TKIs is the ability of lenvatinib to also inhibit FGF-R1, representing an effective drug in those cases in which a resistance to VEGF/VEGF-R inhibitors is developed.

\section{TKRs inhibited by lenvatinib}

Lenvatinib is a multikinase inhibitor, and to better understand its cytostatic activity it is important to better know the physiological activities of the TKRs blocked by the drug. The following is a list and a description of the most important TKRs against which lenvatinib is very active:

1) VEGF-R: It is expressed on the cell membrane of endothelial cells in response to hypoxia, oncogenes, or cytokines. VEGF-R regulates normal and abnormal blood vessel growth; in particular, it regulates both vascular proliferation and permeability and works as an antiapoptotic factor for newly formed blood vessels. VEGF-R expression correlates with poor prognosis in several types of human malignancies. Moreover, VEGF-Rs are related to $c-K I T$ and PDGF-R, which are important in the tumoral development.

2) $P D G F-R$ : The function of VEGF-R in vessel formation is complemented by PDGF-R since its signaling also indirectly regulates angiogenesis. Similar to FGF-R, PDGF-R $\alpha$ signaling has been demonstrated to be necessary for cellular migration. ${ }^{23}$

3) FGF-R: This comprises a family of heparin-binding proteins that currently includes 23 members that signal through four high-affinity TKRs. ${ }^{24}$ Combinations of FGF, FGF-R isoforms, and adaptor proteins lead to signaling networks that play fundamental roles in development, organogenesis, cell differentiation, angiogenesis, and tumor progression. ${ }^{24,25}$

4) RET/PTC: RET is a protooncogene that encodes a TKR involved in cell survival. When a translocation of its $\mathrm{C}$-terminus with various $\mathrm{N}$-terminus genes and their promoters occurs in PTC, different chimeric oncogenes are formed. ${ }^{26,27}$ RET/PTC1 and RET/PTC3 represent 
more than $90 \%$ of all rearrangements, with RET/PTC1 being detected in approximately two-thirds, RET/PTC3 in approximately one-third, ${ }^{28}$ and $R E T / P T C 2$ in less than $5 \%$ of all RET/PTC rearrangements. RET/PTC rearrangements have been shown to signal through $R A S, B-R A F$, and $A k t .^{29,30}$ These genetic alterations are particularly associated with PTC in both patients previously exposed to ionizing radiation and in young adults and children with DTCs. ${ }^{31-34}$

5) $c$-KIT: The proto-oncogene $c-K I T$ is a Type III receptor tyrosine kinase, cellular homologue of the viral oncogene of the feline sarcoma retrovirus HZ4-FeSV. Multiple miRNAs, predicted to target c-KIT, have been reported to be upregulated in PTC, ${ }^{35}$ indicating that the $c$-KIT receptor may be involved in the growth control of thyroid epithelium and that this function may be lost in malignant transformation.

\section{Clinical trials on efficacy of lenvatinib}

Lenvatinib has been tested in several clinical trials that have been conducted both in solid tumors and more specifically on TCs. A detailed description of the most significant studies and their results are reported.

\section{Phase I studies}

To identify the maximum tolerated dose (MTD) and pharmacokinetic profile of lenvatinib (Table 1), a nonrandomized, open-label, Phase I, dose-escalation study was conducted by Boss et al. ${ }^{36}$ The study was also performed to gather preliminary data about the antitumor efficacy of the drug. For this purpose, 82 patients with advanced refractory solid tumors were enrolled: 42 patients had carcinoma (colorectal: 12; renal: eight; gastric: six; pancreatic: four; ovarian: three; esophageal: three; endometrial: two; duodenal: one; breast: one; non-small-cell lung: and one; nasopharyngeal: one),
18/82 had sarcoma, 15/82 had melanoma, 4/82 had mesothelioma, and $3 / 82$ had other types of tumors. Patients received lenvatinib orally at different doses, from 0.2 to $32 \mathrm{mg}$, with subsequent expansion of the MTD dose cohort (25 mg). Seventy-one patients received the study drug on an empty stomach, and eleven patients on a full stomach. Pharmacokinetics assessment revealed that, in the fasted group, lenvatinib was rapidly absorbed with maximum concentrations achieved within 3 hours after administration with dose linear kinetics, while, in the group receiving the drug on a full stomach, the peak plasma concentration was achieved in 5 hours (median values: 2.0 vs 5.0 hours, respectively; $P=0.015$ ).

The most frequent adverse events (AEs) related to lenvatinib were hypertension (40\%), proteinuria (26\%), and gastrointestinal symptoms, such as nausea (37\%), diarrhea (34\%), and stomatitis (32\%). Most of these toxicities were Grade 1 or 2 . Dose modifications were required in 40 patients (49\%), with proteinuria and hypertension being the most common reasons for dose modifications (17\% and 11\%, respectively). Although most of the AEs occurred in patients treated with a lenvatinib daily dose $\geq 12 \mathrm{mg}$, the authors concluded that this drug was well tolerated when administered to patients at doses up to $25 \mathrm{mg} / \mathrm{d}$.

Moreover, in this study, the authors also reported PR in patients diagnosed with renal cell carcinoma and melanoma with a progression-free survival (PFS) of 477 and 217 days, respectively.

MTD, dose-limiting toxicity, pharmacokinetics, pharmacodynamics, and efficacy of lenvatinib in patients with advanced solid tumors (nine colon cancer, seven sarcoma, five non-small-cell lung cancer, and six tumors arising from other sites) were also investigated by Yamada et al. ${ }^{37}$ In this study, lenvatinib dose-escalation was performed and the drug was administered orally twice daily (13 $\mathrm{mg}$ twice a day) in a 2-week-on/1-week-off cycle.

Table I Summary of Phase I studies of lenvatinib

\begin{tabular}{llllll}
\hline Phase & References & $\begin{array}{l}\text { Type of } \\
\text { tumor }\end{array}$ & Adverse events & Best response & MTD \\
\hline I & Boss el al, ${ }^{36}$ & Solid tumors & Hypertension, proteinuria, nausea, diarrhea, stomatitis & Renal cell carcinoma, \\
2012 & & melanoma & 25 mg daily \\
I & Yamada & Solid tumors & Hematuria fatigue, hypertension, increase in aspartate \\
et al, ${ }^{37}$ & & $\begin{array}{l}\text { Colon cancer } \\
\text { transaminase, headache, proteinuria, increase in alanine } \\
\text { transaminase, diarrhea, lactate dehydrogenase increased }\end{array}$ & I3 mg twice a day \\
Hong et al, ${ }^{38}$ & Solid tumors & $\begin{array}{l}\text { Hypertension, proteinuria, fatigue, anorexia, decreased } \\
\text { weight, diarrhea, dysphonia }\end{array}$ & $\begin{array}{l}\text { Melanoma, endometrial, renal, } \\
\text { thyroid, islet cell cancers }\end{array}$ & 10 mg twice a day \\
\hline
\end{tabular}

Abbreviation: MTD, maximum tolerated dose. 
In this study, the most frequently reported treatmentrelated AEs were hematuria (74.1\%), fatigue (70.4\%), hypertension $(66.7 \%)$, increased levels of aspartate transaminase $(63.0 \%)$, headache $(63.0 \%)$, proteinuria $(63.0 \%)$, increased levels of alanine transaminase (55.5\%), diarrhea (55.5\%), and increased levels of lactate dehydrogenase (44.0\%). Five out of 27 patients experienced six serious AEs related or possibly related to study medication, which included hypertension ( 0.5 and $6 \mathrm{mg}$ twice a day), hemorrhage (6 mg twice a day), pneumonia with worsening dyspnea (9 $\mathrm{mg}$ twice a day), and decrease in platelet count ( $9 \mathrm{mg}$ twice a day). A PR was documented in one patient with colon cancer at cycle 4 of lenvatinib $2 \mathrm{mg}$ twice a day, while stable disease (SD) was recorded as best overall response in 21 patients $(84 \%$ of the evaluable patients).

Another Phase I dose-escalation study of lenvatinib ${ }^{38}$ conducted on 77 patients with solid tumors showed that the MTD was $10 \mathrm{mg}$ twice a day, administered continuously. In this study, 43 patients were evaluable for response: $21 \%$ of patients achieved a PR and $56 \%$ patients had a clinical benefit in terms of $\mathrm{PR}+\mathrm{SD} \geq 6$ months.

\section{Phase II study}

The encouraging results of Phase I studies have led to the development of lenvatinib Phase II study in advance RAI-R DTCs (papillary, follicular, or Hürthle cells) patients. ${ }^{39}$ Fifty-eight patients with a disease progression demonstrated by response evaluation criteria in solid tumors (RECIST) during the prior 12 months, including patients who had received prior TKI therapy $(\mathrm{n}=17)$, were enrolled. The primary end point was objective response rate (ORR) by RECIST based on independent imaging review, while PFS and safety evaluation represented the secondary end points. The starting dose of the drug was $24 \mathrm{mg}$ once a day in 28-day cycles and could be reduced if an unmanageable toxicity was observed. Serum circulating cytokines and angiogenic factors levels of 51 patients were also assessed 8 days after beginning lenvatinib.

After a minimum follow-up of 14 months, the ORR was $50 \%$ with several PRs reported. The ORR of patients who had received previous TKI therapy was similar to those patients who had not received such therapy (59\% and 46\%, respectively). The median time to response was 3.6 months and the median response duration was 12.7 months. The median PFS was 12.6 months (95\% confidence interval: 9.9-16.1 months), and also in these cases, the result was similar in patients who received (PFS: 12.2 months) or did not receive (PFS: 12.6 months) prior TKI therapy.
Regarding the levels of circulating cytokines and angiogenic factors in the serum, changes were observed in 16/51 analyzed patients, including increases in levels of placental growth factor and vascular endothelial growth factor $\mathrm{A}$ (VEGF-A), and decreases in levels of angiopoietin-2 (A-2) and soluble vascular endothelial growth factor receptor-2 (sVEGFR-2). Clinical outcomes correlated with levels of cytokine and angiogenic factors levels at baseline, in particular, low baseline levels of A-2 and interleukin-10, and high baseline levels of fms-related tyrosine kinase 3 ligand (an endogenous small molecule that functions as a cytokine and growth factor that increases the number of immune cells) correlated with improved ORR in response to lenvatinib therapy $(P=0.034,0.032$, and 0.041 , respectively). In addition, longer PFS was identified in those patients with low baseline levels of A-2 $(P=0.011)$ and high baseline levels of epidermal growth factor $(P=0.033)$.

All patients experienced treatment-related AEs, which most frequently were hypertension (76\%), weight decrease $(69 \%)$, diarrhea $(67 \%)$, proteinuria $(64 \%)$, fatigue $(60 \%)$, appetite loss (52\%), and nausea (50\%). Most hypertension and proteinuria events were Grade 1 or 2 and were managed without dose adjustments or withdrawal of treatment. Six Grade 4 AEs were reported in five patients including hypocalcemia, hyperkalemia, abasia, and acute myocardial infarction (one report each) and two pulmonary embolism cases. Treatmentrelated AEs led to dose interruptions, reductions, or study drug withdrawal in $74 \%, 66 \%$, and $26 \%$ of patients, respectively.

\section{Phase III study}

A Phase III randomized, double-blind, placebo-controlled study of lenvatinib (the SELECT study) was developed to evaluate the PFS (by independent radiologic review) of subjects with RAI-R DTCs. ${ }^{40}$ The study, which started in August 2011 and completed in October 2012, was an international multicenter randomized study, involving 21 countries all over the world. Main inclusion criterion was disease progression within the prior 13 months and, as in the Phase II study, patients treated with prior TKI therapy were also eligible. Patients of both placebo and lenvatinib groups were stratified according to age, sex, ethnic group, prior treatment or no prior treatment with a TKI, geographic region, histological findings, and baseline characteristics (ie, Eastern Cooperative Oncology Group performance status, presence of lung, or bone metastasis). Two hundred and sixty-one patients were randomly assigned to receive lenvatinib (at a daily dose of $24 \mathrm{mg} / \mathrm{d}$ in 28-day cycles), while 131 patients received placebo. At disease progression, a crossover to open-label 
lenvatinib was available for those patients in therapy with placebo. Dose interruptions and incremental reductions in the dose (to 20,14 , or $10 \mathrm{mg} / \mathrm{d}$ ) due to AEs were permitted. The primary end point was PFS, while secondary end points included the ORR, OS, and safety. In addition, available archival formalin-fixed, paraffin-embedded tissues were obtained and analyzed for $B R A F$ and $R A S$ mutation hotspots.

The median PFS was significantly longer in lenvatinib group than in placebo (18.3 vs 3.6 months; $P<0.001$ ), with a 6-month PFS rate of $77.5 \%$ in the lenvatinib group and $25.4 \%$ in the placebo group. The same results of PFS were observed when a stratification of the group was considered, and in particular no significant difference of PFS was found between patients treated with prior TKI therapy and patients not treated (15.1 vs 18.7 months) nor according to the $B R A F$ and $R A S$ mutational status.

Regarding the secondary end points, lenvatinib group also had a significantly higher ORR compared to placebo group $(64.8 \%$ vs $1.5 \% ; P<0.001)$, with a median time to response of 2.0 months. In particular, complete responses (CR) occurred in four patients $(1.5 \%)$ in the lenvatinib group but in no patients in the placebo group; PR occurred in $63.2 \%$ and in $1.5 \%$, respectively; and SD (durable for 23 weeks or longer) occurred in $15.3 \%$ and $29.8 \%$ of patients, respectively. At the time of the primary analysis, the difference in OS between the groups was not significant, probably due to a potential bias introduced by the crossover phase. However, it has been recently demonstrated that a difference in OS was observed when the analysis was restricted to either the follicular histotype or the group of older patients ( $>65$ years). ${ }^{41,42}$

The incidence of treatment-related AEs (of all grades) was higher in lenvatinib group than in placebo group $(97.3 \%$ vs $59.5 \%$ ). In particular, the incidence of treatment-related AEs of Grade 3 or higher was $75.9 \%$ in the lenvatinib group and $9.9 \%$ in the placebo group. The study confirmed that the most common side effects of this drug are hypertension, occurring in $68 \%$ of patients, diarrhea, decreased appetite, weight loss, nausea, and proteinuria with no unexpected toxicities on the basis of previous Phase I and II studies.

Six out of 20 deaths that occurred in lenvatinib group were considered, by investigators, to be related to study treatment, including one case of pulmonary embolism, one of hemorrhagic stroke, and one of general deterioration of physical health; three cases were reported as deaths or sudden deaths (not otherwise specified).

AEs that led to the discontinuation of treatment were reported in $14.2 \%$ of lenvatinib group and in $3.2 \%$ of placebo group. Among these AEs, the most common were hypertension and asthenia. Interruption or reduction due to AEs were more frequent in lenvatinib than in placebo group (82.4\% vs $18.3 \%$ and $67.8 \%$ vs $4.6 \%$, respectively), resulting in a mean lenvatinib dose of $17.2 \mathrm{mg} / \mathrm{d}$. The most common side effects that required a dose adjustment were diarrhea (22.6\%), hypertension (19.9\%), proteinuria (18.8\%), and decreased appetite (18.0\%).

On the basis of the results of SELECT study, it appears that the majority of AEs due to lenvatinib can be successfully managed by either precociously treating patients, as for hypertension, or by reducing the daily dose to a level that maintains the cytostatic activity avoiding AEs, as for fatigue.

\section{Lenvatinib in MTC and ATC}

Efficacy of lenvatinib was also demonstrated for other histotypes of TCs. In particular, Schlumberger et $\mathrm{al}^{43}$ presented the results of a Phase II study of lenvatinib in advanced unresectable MTC. As with the DTCs study, patients were eligible if progressive disease was confirmed by RECIST in the prior 12 months. A prior VEGFR-targeted therapy was permitted, and during the study, tumor genetic analysis and circulating cytokine and angiogenic factor analysis were performed. As for the Phase II study in DTCs, the primary end point was ORR by RECIST. Fifty-nine patients were enrolled and were treated with a starting dose of lenvatinib $24 \mathrm{mg}$ once daily in 28-day cycles until disease progression or development of unmanageable toxicities. PR was observed in 21 patients (response rate: $36 \%$ ) based on independent imaging review and in 29 patients (response rate: 49\%) based on investigator assessment. The authors found ORR almost similar in the group who received prior VEGFR-directed treatment $(n=26)$ and in the group with no prior VEGFR-directed treatment ( $35 \%$ vs $36 \%$, respectively). At the time of study presentation, median PFS was 9.0 months (based on minimum 8 months of follow-up). Regarding the tumor genetic analysis, there was no clear difference in the response between RET-mutant and $R E T$-wild-type patients.

Recently, lenvatinib antiangiogenesis activity has been also demonstrated in five ATC nude mice in a preclinical study conducted by Tohyama et al. ${ }^{44}$ An international Phase II study of lenvatinib in ATC will likely start in a few months and, hopefully, will answer the question of whether lenvatinib can be successfully used also in ATC.

\section{Other TKIs in DTCs}

In the last decades, since the knowledge about the mechanism of tumors development and spread have been enriched, several oral molecules have been studied as potential targeted therapies for TCs. As listed in Table 2, the majority of TKIs 
Table 2 Studies investigating tyrosine kinase inhibitors in advanced and progressive thyroid cancer

\begin{tabular}{|c|c|c|c|c|c|}
\hline Drug & Phase (reference) & Tumor hystotype & $\begin{array}{l}\text { Median PFS } \\
\text { (months) }\end{array}$ & SD (\%) & RR (\%) \\
\hline \multirow[t]{3}{*}{ Axitinib (AG0I3736) } & I (Rugo et al 2005) $)^{54}$ & NR & NR & NR & NR \\
\hline & II (Cohen et al 2008) $)^{55}$ & DTCs, MTC, ATC & 18.8 & 38 & 30 \\
\hline & II (Locati et al 20I4) $)^{56}$ & DTCs, MTC & 16.1 & 36 & 35 \\
\hline \multirow[t]{3}{*}{ Cabozantinib (XLI84) } & $\mathrm{I}(\text { Kurzrock et al 20I I) })^{57}$ & MTC & NR & 41 & 29 \\
\hline & I (Cabanillas et al 2014) $)^{58}$ & DTCs & NR & 40 & 53 \\
\hline & III (Elisei et al 20I3) $)^{59}$ & MTC & 11.2 & NR & 28 \\
\hline Dovitinib & II (Lim et al 2015) $)^{60}$ & DTCs, MTC & 5.4 & 48.7 & 20.5 \\
\hline \multirow[t]{2}{*}{ Imatinib } & II (De Groot et al 2007)61 & MTC & NR & 27 & 0 \\
\hline & II $(\mathrm{Ha} \text { et al } 2010)^{62}$ & ATC & NR & 50 & 25 \\
\hline \multirow[t]{3}{*}{ Motesanib (AMG706) } & I (Rosen et al 2007) $)^{63}$ & DTCs, MTC, ATC & NR & $43^{\mathrm{a}}$ & $43^{\mathrm{a}}$ \\
\hline & II (Sherman et al 2008) $)^{64}$ & DTCs & 9.2 & 35 & 14 \\
\hline & II (Shlumberger et al 2009) ${ }^{65}$ & MTC & 12 & 48 & 2 \\
\hline \multirow[t]{2}{*}{ Pazopanib } & II (Bible et al 2010) $)^{66}$ & DTCs & 11.7 & NR & 49 \\
\hline & II (Bible et al 20I2) ${ }^{67}$ & ATC & 2 & NR & 0 \\
\hline Selumetinib (AZD6244) & II (Hayes et al 20I2) ${ }^{68}$ & DTCs & 8 & 54 & 3 \\
\hline \multirow[t]{4}{*}{ Sorafenib (BAY43-9006) } & II (Lam et al 20I0) ${ }^{69}$ & MTC & 17.9 & 88 & 6 \\
\hline & II (Schneider et al 20I2)70 & DTCs & 18 & 42 & 31 \\
\hline & II (Savvides et al 2013) & ATC & 1.9 & 25 & 10 \\
\hline & III (Brose et al 20I4) $)^{72}$ & DTCs & 10.8 & 42 & 12 \\
\hline \multirow[t]{2}{*}{ Sunitinib } & II (Carr et al 2010) $)^{73}$ & DTCs, MTC & 12.8 & 46 & 31 \\
\hline & II (Ravaud et al 2008) $)^{74}$ & DTCs, MTC, ATC & NR & 80 & 6 \\
\hline \multirow[t]{3}{*}{ Vandetanib (ZD6474) } & II (Wells et al 2010) ${ }^{75}$ & MTC & 28 & 53 & 20 \\
\hline & III (Wells et al 2012) & MTC & 30.5 & NR & 45 \\
\hline & II (Leuboulleux et al 20I2) $)^{77}$ & DTCs & II.I & NR & 8.3 \\
\hline
\end{tabular}

Notes: aPercentage referred to seven cases of thyroid cancer (study conducted on different type of solid tumor).

Abbreviations: DTCs, differentiated thyroid cancers; MTC, medullary thyroid cancer; ATC, anaplastic thyroid cancer; PFS, progression free survival; SD, stable disease; RR, response rate; NR, not reached or not reported.

were tested in different histotypes of TCs. At the moment, in addition to lenvatinib, Phase III studies on DTCs patients are designed only for sorafenib and vandetanib (the latter still ongoing).

\section{Sorafenib (BAY 43-9006, Nexavar)}

This is an oral small molecule that is able to inhibit $C-R A F$, $B-R A F, R E T, c-K I T, P D G F-R$, and VEGF-R 1-3. A multicenter, randomized, double-blind, placebo-controlled, Phase III trial (DECISION study) has led to the approval of sorafenib for the treatment of well-differentiated RAI-R metastatic DTCs. ${ }^{45}$ The DECISION study was conducted on the basis of several Phase II studies, which had shown promising response rates and median PFS in patients with RAI-R DTCs. The main inclusion criterion of DECISION study was progressive disease according to RECIST within the prior 14 months, and no patients who had prior TKI therapy were admitted. To assess PFS (primary end point), 416 patients (207 in the sorafenib group and 209 in the placebo group) were enrolled.

The median PFS was significantly longer in sorafenib group than in placebo group (10.8 vs 5.8 months; $P<0.0001)$.
AEs, most of which were Grade 1 or 2, occurred in $98.6 \%$ of patients receiving sorafenib and in $87.6 \%$ of patients receiving placebo. The most frequent AEs related to this drug were hand and foot skin reaction (76.3\%), diarrhea (68.6\%), alopecia $(67.1 \%)$, and rash or desquamation (50.2\%).

\section{Vandetanib (ZD6474, Caprelsa)}

This is an oral small molecule that mainly inhibits $R E T$, $V E G F-R$ 1-2, and $E G F-R$. At the moment, this drug has been approved for the treatment of advanced and metastatic MTC after a Phase III study (ZETA study) ${ }^{46}$ that demonstrated its efficacy in determining a significant prolongation of the PFS of patients treated with the drug with respect to those treated with placebo. Because of its anti-RET activity, its efficacy was also investigated in patients with locally advanced or metastatic DTCs in a Phase II trial. ${ }^{47}$ In this trial, 72 patients received vandetanib and 73 placebo. An advantage in median PFS was noted in the vandetanib arm (11.1 vs 5.9 months; $P=0.008$ ). The most common Grade 3 or worse AEs were QTc prolongation (14\%), diarrhea (10\%), asthenia (7\%), and fatigue (5\%). Two patients in the vandetanib group and one in the placebo group died from treatment-related serious AEs (hemorrhage 
Table 3 Main differences between the two drugs approved for the treatment of metastatic radioiodine refractory differentiated thyroid cancer: lenvatinib and sorafenib

\begin{tabular}{|c|c|c|c|c|c|c|c|c|c|}
\hline $\begin{array}{l}\text { Drug study } \\
\text { name }\end{array}$ & Daily dose & $\begin{array}{l}\text { Enrolled } \\
\text { subjects }\end{array}$ & Prior TKI & PFS (months) & SD (\%) & CR (\%) & PR (\%) & $\begin{array}{l}\text { Dose reduction or } \\
\text { interruption }(\%)^{\mathrm{a}}\end{array}$ & $\begin{array}{l}\text { Number } \\
\text { of deaths }\end{array}$ \\
\hline Lenvatinib & $24 \mathrm{mg} \times \mathrm{I}$ & 392 & Yes & 18.3 vs $3.6^{b}$ & 15.3 & 1.5 & 63.2 & $78.5 ; 14.2$ & 6 \\
\hline \multicolumn{10}{|l|}{ SELECT } \\
\hline Sorafenib & $400 \mathrm{mg} \times 2$ & 416 & No & 10.8 vs $5.8^{\mathrm{b}}$ & 42 & 0 & 12.2 & $77.8 ; 18.8$ & I \\
\hline DECISION & & & & & & & & & \\
\hline
\end{tabular}

Notes: aDue to AEs; bdrug vs placebo; 'drug related.

Abbreviations: TKI, tyrosine kinase inhibitor; PFS, progression free survival; SD, stable disease; CR, complete response; PR, partial response; AEs, adverse events.

from skin metastases and pneumonia in the vandetanib arm and pneumonia in the placebo arm). A Phase III study of vandetanib in advanced RAI-R DTCs is currently ongoing. This is a multicenter, double-blind, placebo-controlled clinical trial (VERIFY study; unpublished data) that enrolled 255 patients with progressive RAI-R DTCs. The enrollment has been recently completed and data should be soon available.

\section{Therapeutic options in DTCs: lenvatinib vs sorafenib}

At the moment, sorafenib and lenvatinib are the only targeted therapies approved by US Food and Drug Administration (FDA) and European Medical Agency (EMA) for the treatment of RAI-R advanced DTCs patients. As listed in Table 3, some differences are present regarding both the Phase III study designs and the final results.

Two main differences between the two studies are: 1) prior TKI therapy was allowed in lenvatinib but not in sorafenib study; and 2) the progression was assessed centrally by a reviewing board in the SELECT study while it was evaluated by local principal investigators in the DECISION study.

Since in the SELECT study the PFS was demonstrated to be the same in patients priorly treated with TKI and in those naïve to any therapy, this means that lenvatinib may be also used as second-line treatment with a high efficacy in prolonging PFS. This aspect is particularly important for those patients treated with a TKI who develop the so-called "escape phenomenon" after which the tumor, whose growth was controlled for several months, starts to grow again. Although the mechanisms underlying the escape phenomenon are still unknown, it is likely due to the development of a drug resistance determined by both the activation or upregulation of alternative proangiogenic signaling pathways and the selective pressures of the microenvironment during malignant progression. ${ }^{48-50}$ After the escape, only the administration of an other TKI with other mechanisms of action can revert the trend of growth, and lenvatinib has been shown to have such ability in patients who were previously treated with other TKIs. At present, we do not have data on the possibility that sorafenib can act as a second line in patients who develope resistance to other TKIs.

Lenvatinib showed a higher PFS than sorafenib (18.3 vs 10.8 months) and some cases of CR (1.5\% vs $0 \%)$. In addition, the percentage of PR was higher in lenvatinib than in sorafenib-treated patients (63.2\% vs $12.2 \%$ ). However, a higher number of deaths related to study drug was described for lenvatinib than sorafenib. Although this high number of deaths was reported to be related to lenvatinib use $(n=6)$, four of these deaths were nonspecific in their cause (sudden death, general deterioration, and two unspecified), and the relation to the drug was established by the interpretation of different investigators. In this regard, it is relevant to underline that patients enrolled in the SELECT study were affected by a more severe disease, as demonstrated by the shorter PFS observed in the placebo arm of SELECT study with respect to the PFS in the placebo arm of DECISION study (3.6 vs 5.8 months). The difference in the severity of the disease in the two populations could have somehow played a role in the outcome of these patients.

As far as the AEs impact on the management of the two drugs is concerned, it is worth noting that no major differences were found between the two drugs regarding the percentage of dose reductions and interruptions due to AEs.

\section{Major concerns}

Although both lenvatinib and sorafenib are at present the best treatment options for RAI-R DTCs patients, there are some general concerns related to their long-term use. As mentioned earlier, they are mainly cytostatic drugs and their administration should be continued until the evidence of clinical benefit. This concept could imply a lifelong treatment, and in cases with unmanageable AEs the patients' quality of life can be greatly affected. A cost-benefit evaluation between the quality of life and disease control, especially in cases with a low growth rate, should be considered at the time of taking the decision to start the therapy. 
Another aspect to take into consideration is that, until now, the duration of the TKI response is not durable and escape phenomenon will arrive sooner or later. We have stated that lenvatinib showed efficacy in prolonging PFS also in patients previously treated with other TKIs $;{ }^{40}$ thus, the possibility to use TKIs in second and third line is an option. However, new drugs, already tested in other human tumors ${ }^{51}$ and acting by improving inflammatory response against the tumoral tissue, are under investigation also for the treatment of TCs either as single agent or in combination with other TKIs (ClinicalTrials.gov Identifier: NCT02501096).

\section{Conclusion}

Lenvatinib represents a new option for the treatment of RAI-R advanced DTCs patients that is able to inhibit multiple TKRs and the process of angiogenesis. A recent Phase III study has demonstrated its efficacy in prolonging PFS in patients with advanced DTCs and in achieving a high clinical benefit $(65 \%)$ with an acceptable toxicity profile. Since hypertension was the most common side effect, it should be considered that, as previously demonstrated, ${ }^{52,53}$ an antihypertension medication and dose reduction are usually able to keep the majority of patients on treatment with lenvatinib. Nonetheless, the balance is still unclear between the clinical benefits and the quality of life due to other AEs and further assessments are needed to establish the real incidence of fatal events.

Since it is well established that maintaining a patient on a TKI as long as possible can maximize tumor response, lenvatinib, as well as the majority of TKI drugs, should be managed by experts able to recognize early the beginning of AEs before they become unmanageable and before they require a dose interruption. A good experience in the treatment of TCs and particularly of advanced TCs is also fundamental.

\section{Disclosure}

Rossella Elisei is a consultant and a speaker for Genzyme, Eisai, Exelixis, Bayer. The authors declare that this review is an independent work and expression of personal experience and knowledge. The authors report no other conflicts of interest in this work.

\section{References}

1. National Cancer Institute. Surveillance epidemiology and end results program: seer stat facts: thyroid cancer. Available from: http://seer. cancer.gov/statfacts/html/thyro.html (cited 2015 December 29). Accessed August 1, 2015.

2. Schlumberger MJ. Papillary and follicular thyroid carcinoma. $N$ Engl J Med. 1998;338(5):297-306.

3. Biondi B, Cooper DS. Benefits of thyrotropin suppression versus the risks of adverse effects in differentiated thyroid cancer. Thyroid. 2010;20(2):135-146.
4. Elisei R, Pinchera A, Chiovato L, et al. Transfection with the cDNA of the human thyrotropin receptor of a poorly differentiated rat thyroid cell line (FRT). J Endocrinol Invest. 1996;19(4):230-235.

5. Elisei R, Molinaro E, Agate L, et al. Are the clinical and pathological features of differentiated thyroid carcinoma really changed over the last 35 years? Study on 4187 patients from a single Italian institution to answer this question. J Clin Endocrinol Metab. 2010;95(4):1516-1527.

6. Eustatia-Rutten CF, Corssmit EP, Biermasz NR, Pereira AM, Romijn JA, Smit JW. Survival and death causes in differentiated thyroid carcinoma. J Clin Endocrinol Metab. 2006;91(1):313-319.

7. Durante C, Haddy N, Baudin E, et al. Long-term outcome of 444 patients with distant metastases from papillary and follicular thyroid carcinoma: benefits and limits of radioiodine therapy. J Clin Endocrinol Metab. 2006;91(8):2892-2899.

8. Haugen BRM, Alexander EK, Bible KC, et al. 2015 American Thyroid Association Management Guidelines for adult patients with thyroid nodules and differentiated thyroid cancer. Thyroid. 2016;26(1):1-33.

9. Kim BM, Kim MJ, Kim EK, Park SI, Park CS, Chung WY. Controlling recurrent papillary thyroid carcinoma in the neck by ultrasonographyguided percutaneous ethanol injection. Eur Radiol. 2008;18(4): $835-842$.

10. Baek JH, Kim YS, Lee D, Huh JY, Lee JH. Benign predominantly solid thyroid nodules: prospective study of efficacy of sonographically guided radiofrequency ablation versus control condition. AJR Am J Roentgenol. 2010;194(4):1137-1142.

11. Papini E, Bizzarri G, Bianchini A, et al. Percutaneous ultrasound-guided laser ablation is effective for treating selected nodal metastases in papillary thyroid cancer. J Clin Endocrinol Metab. 2013;98(1):E92-E97.

12. Park KW, Shin JH, Han BK, Ko EY, Chung JH. Inoperable symptomatic recurrent thyroid cancers: preliminary result of radiofrequency ablation. Ann Surg Oncol. 2011;18(9):2564-2568.

13. Sherman SI, Wirth LJ, Droz JP, et al. Motesanib diphosphate in progressive differentiated thyroid cancer. $N$ Engl J Med. 2008;359(1):31-42.

14. Krause DS, Van Etten RA. Tyrosine kinases as targets for cancer therapy. N Engl J Med. 2005;353(2):172-187.

15. Antonelli A, Fallahi P, Ferrari SM, et al. Dedifferentiated thyroid cancer: a therapeutic challenge. Biomed Pharmacother. 2008;62(8):559-563.

16. Nikiforova MN, Nikiforov YE. Molecular genetics of thyroid cancer: implications for diagnosis, treatment and prognosis. Expert Rev Mol Diagn. 2008;8(1):83-95.

17. Kroll TG, Sarraf P, Pecciarini L, et al. PAX8-PPARgamma1 fusion oncogene in human thyroid carcinoma [corrected]. Science. 2000; 289(5483):1357-1360.

18. Soh EY, Duh QY, Sobhi SA, et al. Vascular endothelial growth factor expression is higher in differentiated thyroid cancer than in normal or benign thyroid. J Clin Endocrinol Metab. 1997;82(11):3741-3747.

19. Zhang J, Wang P, Dykstra M, et al. Platelet-derived growth factor receptor-alpha promotes lymphatic metastases in papillary thyroid cancer. J Pathol. 2012;228(2):241-250.

20. Matsui J, Yamamoto Y, Funahashi Y, et al. E7080, a novel inhibitor that targets multiple kinases, has potent antitumor activities against stem cell factor producing human small cell lung cancer H146, based on angiogenesis inhibition. Int J Cancer. 2008;122(3):664-671.

21. Bruheim S, Kristian A, Uenaka T, et al. Antitumour activity of oral E7080, a novel inhibitor of multiple tyrosine kinases, in human sarcoma xenografts. Int J Cancer. 2011;129(3):742-750.

22. Ikuta K, Yano S, Trung VT, et al. E7080, a multi-tyrosine kinase inhibitor, suppresses the progression of malignant pleural mesothelioma with different proangiogenic cytokine production profiles. Clin Cancer Res. 2009;15(23):7229-7237.

23. Glen H, Mason S, Patel H, Macleod K, Brunton VG. E7080, a multitargeted tyrosine kinase inhibitor suppresses tumor cell migration and invasion. BMC Cancer. 2011;11:309.

24. Ezzat S, Asa SL. FGF receptor signaling at the crossroads of endocrine homeostasis and tumorigenesis. Horm Metab Res. 2005;37(6):355-360.

25. Grose R, Dickson C. Fibroblast growth factor signaling in tumorigenesis. Cytokine Growth Factor Rev. 2005;16(2):179-186. 
26. Santoro M, Carlomagno F, Melillo RM, Billaud M, Vecchio G, Fusco A. Molecular mechanisms of RET activation in human neoplasia. $J$ Endocrinol Invest. 1999;22(10):811-819.

27. Castellone MD, Santoro M. Dysregulated RET signaling in thyroid cancer. Endocrinol Metab Clin North Am. 2008;37(2):363-374, viii.

28. Tallini G, Asa SL. RET oncogene activation in papillary thyroid carcinoma. Adv Anat Pathol. 2001;8(6):345-354.

29. Salvatore G, Chiappetta G, Nikiforov YE, et al. Molecular profile of hyalinizing trabecular tumours of the thyroid: high prevalence of RET/ PTC rearrangements and absence of B-raf and N-ras point mutations. Eur J Cancer. 2005;41(5):816-821.

30. Freche B, Guillaumot $\mathrm{P}$, Charmetant J, et al. Inducible dimerization of RET reveals a specific AKT deregulation in oncogenic signaling. J Biol Chem. 2005;280(44):36584-36591.

31. Favus MJ, Schneider AB, Stachura ME, et al. Thyroid cancer occurring as a late consequence of head-and-neck irradiation. Evaluation of 1056 patients. N Engl J Med. 1976;294(19):1019-1025.

32. Schneider AB, Ron E, Lubin J, Stovall M, Gierlowski TC. Doseresponse relationships for radiation-induced thyroid cancer and thyroid nodules: evidence for the prolonged effects of radiation on the thyroid. J Clin Endocrinol Metab. 1993;77(2):362-369.

33. Fenton CL, Lukes Y, Nicholson D, Dinauer CA, Francis GL, Tuttle RM. The ret/PTC mutations are common in sporadic papillary thyroid carcinoma of children and young adults. J Clin Endocrinol Metab. 2000;85(3):1170-1175.

34. Romei C, Ciampi R, Elisei R. A comprehensive overview of the role of the RET proto-oncogene in thyroid carcinoma. Nat Rev Endocrinol. 2016;12(4):192-202.

35. Mazeh H, Mizrahi I, Halle D, et al. Development of a microRNA-based molecular assay for the detection of papillary thyroid carcinoma in aspiration biopsy samples. Thyroid. 2011;21(2):111-118.

36. Boss DS, Glen H, Beijnen JH, et al. A phase I study of E7080, a multitargeted tyrosine kinase inhibitor, in patients with advanced solid tumours. Br J Cancer. 2012;106(10):1598-1604.

37. Yamada K, Yamamoto N, Yamada Y, et al. Phase I dose-escalation study and biomarker analysis of E7080 in patients with advanced solid tumors. Clin Cancer Res. 2011;17(8):2528-2537.

38. Hong DS, Koetz BS, Kurzrock R, et al. Phase I dose-escalation study of E7080, a selective tyrosine kinase inhibitor, administered orally to patients with solid tumours. Poster presented at: 2010 ASCO Annual Meeting; 2010.

39. Cabanillas ME, Schlumberger M, Jarzab B, et al. A phase 2 trial of lenvatinib (E7080) in advanced, progressive, radioiodine-refractory, differentiated thyroid cancer: a clinical outcomes and biomarker assessment. Cancer. 2015;121(16):2749-2756.

40. Schlumberger M, Tahara M, Wirth LJ, et al. Lenvatinib versus placebo in radioiodine-refractory thyroid cancer. $N$ Engl J Med. 2015;372(7): 621-630.

41. Brose MS, Tahara M, Wirth LJ, et al. Effect of age and lenvatinib treatment on overall survival for patients with 131I-refractory differentiated thyroid cancer in SELECT. Poster Session 2015 ASCO Annual Meeting; 2015.

42. Elisei R, Schlumberger MJ, Tahara M, et al. Subgroup analysis according to differentiated thyroid cancer histology in phase 3 (SELECT) trial of lenvatinib. Oncol Res Treat 2015. 2015;38 (suppl 5):1-270, abstract 91.

43. Schlumberger M, Carlomagno F, Baudin E, Bidart JM, Santoro M. New therapeutic approaches to treat medullary thyroid carcinoma. Nat Clin Pract Endocrinol Metab. 2008;4(1):22-32.

44. Tohyama O, Matsui J, Kodama K, et al. Antitumor activity of lenvatinib (e7080): an angiogenesis inhibitor that targets multiple receptor tyrosine kinases in preclinical human thyroid cancer models. J Thyroid Res. 2014;2014:638747.

45. Brose MS, Nutting CM, Jarzab B, et al. Sorafenib in radioactive iodine-refractory, locally advanced or metastatic differentiated thyroid cancer: a randomised, double-blind, phase 3 trial. Lancet. 2014; 384(9940):319-328.
46. Wells SA Jr, Robinson BG, Gagel RF, et al. Vandetanib in patients with locally advanced or metastatic medullary thyroid cancer: a randomized, double-blind phase III trial. J Clin Oncol. 2012;30(2):134-141.

47. Leboulleux S, Bastholt L, Krause T, et al. Vandetanib in locally advanced or metastatic differentiated thyroid cancer: a randomised, double-blind, phase 2 trial. Lancet Oncol. 2012;13(9):897-905.

48. Bergers G, Hanahan D. Modes of resistance to anti-angiogenic therapy. Nat Rev Cancer. 2008;8(8):592-603.

49. Miller KD, Sweeney CJ, Sledge GW Jr. Can tumor angiogenesis be inhibited without resistance? EXS. 2005(94):95-112.

50. Kerbel RS, Yu J, Tran J, et al. Possible mechanisms of acquired resistance to anti-angiogenic drugs: implications for the use of combination therapy approaches. Cancer Metastasis Rev. 2001;20(1-2):79-86.

51. Carbognin L, Pilotto S, Milella M, et al. Differential activity of nivolumab, pembrolizumab and MPDL3280A according to the tumor expression of programmed death-ligand-1 (PD-L1): sensitivity analysis of trials in melanoma, lung and genitourinary cancers. PLoS One. 2015; 10(6):e0130142.

52. Keizer RJ, Gupta A, Shumaker R, Beijnen JH, Schellens JH, Huitema AD. Model-based treatment optimization of a novel VEGFR inhibitor. Br J Clin Pharmacol. 2012;74(2):315-326.

53. Keizer RJ, Gupta A, Mac Gillavry MR, et al. A model of hypertension and proteinuria in cancer patients treated with the anti-angiogenic drug E7080. J Pharmacokinet Pharmacodyn. 2010;37(4):347-363.

54. Rugo HS, Herbst RS, Liu G, et al. Phase I trial of the oral antiangiogenesis agent AG-013736 in patients with advanced solid tumors: pharmacokinetic and clinical results. J Clin Oncol. 2005;23(24):5474-5483.

55. Cohen EE, Rosen LS, Vokes EE, et al. Axitinib is an active treatment for all histologic subtypes of advanced thyroid cancer: results from a phase II study. J Clin Oncol. 2008;26(29):4708-4713.

56. Locati LD, Licitra L, Agate L, et al. Treatment of advanced thyroid cancer with axitinib: Phase 2 study with pharmacokinetic/pharmacodynamic and quality-of-life assessments. Cancer. 2014;120(17):2694-2703.

57. Kurzrock R, Sherman SI, Ball DW, et al. Activity of XL184 (Cabozantinib), an oral tyrosine kinase inhibitor, in patients with medullary thyroid cancer. J Clin Oncol. 2011;29(19):2660-2666.

58. Cabanillas ME, Brose MS, Holland J, Ferguson KC, Sherman SI. A phase I study of cabozantinib (XL184) in patients with differentiated thyroid cancer. Thyroid. 2014;24(10):1508-1514.

59. Elisei R, Schlumberger MJ, Muller SP, et al. Cabozantinib in progressive medullary thyroid cancer. J Clin Oncol. 2013;31(29):3639-3646.

60. Lim SM, Chung WY, Nam KH, et al. An open label, multicenter, phase II study of dovitinib in advanced thyroid cancer. Eur J Cancer. 2015;51(12):1588-1595.

61. de Groot JW, Zonnenberg BA, van Ufford-Mannesse PQ, et al. A phase II trial of imatinib therapy for metastatic medullary thyroid carcinoma. J Clin Endocrinol Metab. 2007;92(9):3466-3469.

62. Ha HT, Lee JS, Urba S, et al. A phase II study of imatinib in patients with advanced anaplastic thyroid cancer. Thyroid. 2010;20(9):975-980.

63. Rosen LS, Kurzrock R, Mulay M, et al. Safety, pharmacokinetics, and efficacy of AMG 706, an oral multikinase inhibitor, in patients with advanced solid tumors. J Clin Oncol. 2007;25(17):2369-2376.

64. Sherman SI, Wirth LJ, Droz JP, et al. Motesanib diphosphate in progressive differentiated thyroid cancer. $N$ Engl J Med. 2008;359(1): $31-42$.

65. Schlumberger MJ, Elisei R, Bastholt L, et al. Phase II study of safety and efficacy of motesanib in patients with progressive or symptomatic, advanced or metastatic medullary thyroid cancer. J Clin Oncol. 2009;27(23):3794-3801.

66. Bible KC, Suman VJ, Molina JR, et al. Efficacy of pazopanib in progressive, radioiodine-refractory, metastatic differentiated thyroid cancers: results of a phase 2 consortium study. Lancet Oncol. 2010;11(10):962-972.

67. Bible KC, Suman VJ, Menefee ME, et al. A multiinstitutional phase 2 trial of pazopanib monotherapy in advanced anaplastic thyroid cancer. J Clin Endocrinol Metab. 2012;97(9):3179-3184. 
68. Hayes DN, Lucas AS, Tanvetyanon T, et al. Phase II efficacy and pharmacogenomic study of Selumetinib (AZD6244; ARRY-142886) in iodine-131 refractory papillary thyroid carcinoma with or without follicular elements. Clin Cancer Res. 2012;18(7):2056-2065.

69. Lam ET, Ringel MD, Kloos RT, et al. Phase II clinical trial of sorafenib in metastatic medullary thyroid cancer. J Clin Oncol. 2010;28(14):2323-2330.

70. Schneider TC, Abdulrahman RM, Corssmit EP, Morreau H, Smit JW, Kapiteijn E. Long-term analysis of the efficacy and tolerability of sorafenib in advanced radio-iodine refractory differentiated thyroid carcinoma: final results of a phase II trial. Eur J Endocrinol. 2012;167(5):643-650.

71. Savvides P, Nagaiah G, Lavertu P, et al. Phase II trial of sorafenib in patients with advanced anaplastic carcinoma of the thyroid. Thyroid. 2013;23(5):600-604.

72. Brose MS, Nutting CM, Jarzab B, et al. Sorafenib in radioactive iodine-refractory, locally advanced or metastatic differentiated thyroid cancer: a randomised, double-blind, phase 3 trial. Lancet. 2014;384(9940):319-328.
73. Carr LL, Mankoff DA, Goulart BH, et al. Phase II study of daily sunitinib in FDG-PET-positive, iodine-refractory differentiated thyroid cancer and metastatic medullary carcinoma of the thyroid with functional imaging correlation. Clin Cancer Res. 2010;16(21):5260-5268.

74. Ravaud A, Wallerand H, Culine S, et al. Update on the medical treatment of metastatic renal cell carcinoma. Eur Urol. 2008;54(2):315-325.

75. Wells SA Jr, Gosnell JE, Gagel RF, et al. Vandetanib for the treatment of patients with locally advanced or metastatic hereditary medullary thyroid cancer. J Clin Oncol. 2010;28(5):767-772.

76. Wells SA Jr, Robinson BG, Gagel RF, et al. Vandetanib in patients with locally advanced or metastatic medullary thyroid cancer: a randomized, double-blind phase III trial. J Clin Oncol. 2012;30(2):134-141.

77. Leboulleux S, Bastholt L, Krause T, et al. Vandetanib in locally advanced or metastatic differentiated thyroid cancer: a randomised, double-blind, phase 2 trial. Lancet Oncol. 2012;13(9):897-905.
OncoTargets and Therapy

\section{Publish your work in this journal}

OncoTargets and Therapy is an international, peer-reviewed, open access journal focusing on the pathological basis of all cancers, potential targets for therapy and treatment protocols employed to improve the management of cancer patients. The journal also focuses on the impact of management programs and new therapeutic agents and protocols on

\section{Dovepress}

patient perspectives such as quality of life, adherence and satisfaction. The manuscript management system is completely online and includes a very quick and fair peer-review system, which is all easy to use. Visit http://www.dovepress.com/testimonials.php to read real quotes from published authors. 